\title{
Thermodynamics of the Bose-system with a small number of particles
}

\author{
I.O.Vakarchuk, A.A.Rovenchak \\ Department for Theoretical Physics of Lviv National University, \\ 12 Drahomanov Str., 79005, Lviv, Ukraine
}

Received May 28, 2001

\begin{abstract}
A theoretical description of the interacting Bose-system is proposed. It is based on the extrapolation of the results obtained for the systems with a small number of particles $N=2,3,4$, etc. to the bulk case of $N=\infty$. It is shown that already the system with $N=12,13$ behaves almost as a bulk in a wide temperature range. Special attention is paid to the phase transition in these systems. The hard sphere potential is used in calculations. The sequence of heat capacity maxima is approximated as $C_{N}^{\max } / N \simeq 13.6-a N^{-\varepsilon}$ with $\varepsilon=0.0608$ giving the value of bulk heat capacity as 13.6 while experimental value is close to 16 . The temperature of $\lambda$-transition is estimated as $2.1-2.3 \mathrm{~K}$ versus experimental $2.17 \mathrm{~K}$. Quite good qualitative and satisfactory quantitative agreement with the experimental data has been achieved.
\end{abstract}

Key words: Boson systems, finite systems, lambda-transition, specific heat

PACS: 05.30.Jp, 74.25.Bt

\section{Introduction}

Liquid ${ }^{4} \mathrm{He}$ is the only atomic system having its properties determined mainly by quantum effects and the most essential among the latter is the statistical effect of identical particles. Due to it the macroscopic number of the ${ }^{4} \mathrm{He}$ atoms can have a zero momentum at some conditions, i. e., it can occupy a single quantum state. The phenomenon of bosons accumulation at the lowest energy level is called Bose-Einstein condensation (1924). According to London's assumption (1938), it is believed that superfluidity, $\lambda$-transition, and other unusual properties of liquid ${ }^{4} \mathrm{He}$ are a consequence of this very effect.

However, despite the lapse of time that has passed since the discovery of the phenomena of $\lambda$-transition by Keesom and Clausius in 1932 and the superfluidity by Kapitsa in 1938, nobody has managed to create a comprehensive microscopic theory explaining the properties of strongly interacting Bose-systems such as ${ }^{4} \mathrm{He}$ 
within the whole temperature range of $0<T<\infty$.

Bogoliubov [1] was the first to show how one can build a theory of real helium proceeding from the approximate second quantization techniques for the model of weakly-imperfect Bose-gas. This theory works well for $T<1 \mathrm{~K}$, especially while $T \rightarrow 0$.

The subsequent theoretical studies brought about the appearance of the quantitative theory of the ground state of a strongly-imperfect many-boson system leading to a good agreement with the experiment and to a theoretical description of lowenergy excitations which are responsible for the low-temperature behaviour of thermodynamic functions. The experimental investigations of the spatial structure by means of X-ray and neutron diffraction and of the structure of the energy spectrum and the condensate fraction by means of the inelastic neutron scattering revealed a fairly good theoretical explanation.

Proceeding from of the experimental evidence, it was supposed for a long time that ${ }^{4} \mathrm{He}$ heat capacity had a logarithmic divergency at the transition temperature $T_{\lambda}$. Brout showed in his early works [2] based on the Hartree-Fock method that it was impossible to find an explanation of the observed heat capacity behaviour within simple theoretical approaches. Feynman made an interesting notice regarding this issue in his book [3]: "The explanation of this behaviour is left as an exercise for the reader. If successful, publish!"

Feynman suggested that the $\lambda$-transition should be studied using the full $N$ particle density matrix for the ideal Bose-gas with a phenomenologically introduced effective mass for particles [4]. This approach leads only to a shift of the Bosecondensation temperature in comparison with the ideal case leaving the form of the heat capacity curve unchanged.

In the early 1970s the studies of the $\lambda$-transition were started in the renormalization group (RG) approach for the theory with the degenerate two-component order parameter. The rigorous grounds of this approach based on the functional integration techniques are given in $[5,6]$. They use the coherent states representation with the complex order parameter the absolute value of which equals $\sqrt{N_{0}}$ where $N_{0}$ is the number of atoms in Bose-condensate.

In this approach, the heat capacity critical exponent was calculated as $\alpha=1 / 8$. It obviously does not correspond to the logarithmic divergence for which $\alpha$ equals zero. However, taking into account that the calculated value of $\alpha$ is a small magnitude, the experimental confirmation of this result required very precise measurements which became available only recently [7]. Before it, there was even no certainty about the sign of $\alpha$. The measurements showed that the critical exponent $\alpha$ is negative, i. e., the heat capacity is finite at the transition temperature.

Recently this result was theoretically confirmed. Kleinert [8,9] obtained for $\alpha$ the respective values of $\alpha=-0.01294 \pm 0.00060$ and $\alpha=-0.0120 \pm 0.0009$, while the experiment gives $\alpha=-0.01285 \pm 0.00038$ [7]. One can find a bit different approach to the critical exponents calculation in the paper by Campostrini et al. [10]. The authors obtained the value of $\alpha=-0.0150 \pm 0.0017$. But, despite a very good agreement of these results with the experiment, RG works only in the immediate 
vicinity of the transition point $T_{\lambda}$ and gives no possibility of getting some information of the thermodynamic functions behaviour in a wider near-critical range, e. g., for $\left|T-T_{\lambda}\right| \sim 10^{-3} \mathrm{~K}$.

In addition to these theoretical investigations, the numerical studies of ${ }^{4} \mathrm{He}$ thermodynamic properties were conducted. The Diffusion Monte Carlo (DMC) techniques are applied for the ground state calculations, and the Path Integral Monte Carlo (PIMC) is used for finite temperatures. These techniques have been applied since the 1960s. At present, PIMC is the most effective method. Its sole and essential imperfection lies in the fact that it is impossible to use it for the ground state $(T=0 \mathrm{~K})$. But one can avoid this problem by considering the limit of the results at $T \rightarrow 0 \mathrm{~K}$. A detailed analysis of PIMC might be found in the work by Ceperley [11]. It appears that the numerical approach provides a good agreement with the experimental data in a wide temperature range on the whole, except for the vicinity of the $\lambda$-transition point.

Thus, we have the situation when no satisfactory theoretical description of ${ }^{4} \mathrm{He}$ exists for a considerable temperature range of $1 \mathrm{~K} T<T_{\lambda}$ and $T>T_{\lambda}$, and the only way out is the application of numerical techniques. It gives some space for the investigations in this domain. Not claiming the solution of the problem, we still propose here the model which, in our opinion, can near us to the understanding of the processes in many-boson system via the consideration of systems with a small number of particles.

For the latter, one can expect a much more precise description in terms of statistical mechanics in comparison with what might be reached while directly considering a many-particle system.

The aim of the present work consists in obtaining a thermodynamic description of an interacting many-boson system based on the results for the systems with a small number of particles $N$. We start our analysis from $N=2,3,4$, etc. in order to extrapolate these results for the case of $N=\infty$ after establishing some dependencies on $N$ and the temperature. We pay a special attention to the fact that even the system consisting of a small number of particles exhibits the peculiarities on the heat capacity curve such as the maximum which means the phase transition in the limit of $N \rightarrow \infty$. We will show at the same time that the system with $N=12,13$ tends to reveal qualitatively an "almost bulk" behaviour in a wide temperature range.

The boson systems with a relatively small number of particles have been experimentally obtained only in the last couple of years as particles in traps (see $[12,13]$ and references therein). It enables us to develop some methods for such systems and a further comparison of the results with the experimental data.

We use the partition function formalism to calculate thermodynamic characteristics of the free Bose-system. A method somewhat similar to this one was used in works $[12,13]$ for the study of the trapped finite Bose-systems: the recursion formulae for the partition function proposed by P.Borrmann and G.Franke [14]. But our expressions do not take the restriction for the system energy to be written as a sum of one-particle energies. So, one can use them not only for the ideal gas but for the 
interacting system as well.

The paper is organized as follows. In section 2 some known initiating expressions for calculating the thermodynamic function are given in short. In sections 3 and 4 we consider how the proposed method works in the case of an ideal Bose-gas the results for which are well-known in the thermodynamic limit when the number of particles $N \rightarrow \infty$. The next step is to determine the asymptotic behaviour of our results depending on the number of particles $N$. We will try to expand the found regularities for the case of interacting bosons. The corresponding calculations for the hard-sphere potential are given in section 5 .

Generally, it is impossible to obtain a non-analyticity point of the heat capacity function having a finite number of particles. Therefore, no "pure" phase transition can be detected in this model. But we expect a certain qualitative agreement with the experiment to be quite sufficient for the approximation used.

\section{General statements}

We consider the system of $N$ particles with the Hamiltonian $\hat{H}_{N}$. The partition function $Z_{N}=\operatorname{Spe~e}^{-\beta \hat{H}_{N}}$ where $\beta$ is inverse temperature, $\beta=1 / T$.

In the coordinate representation we have

$$
Z_{N}=\int \mathrm{d} \mathbf{r}_{1} \ldots \int \mathrm{d} \mathbf{r}_{N} R_{N}\left(\mathbf{r}_{1}, \ldots, \mathbf{r}_{N} \mid \mathbf{r}_{1}, \ldots, \mathbf{r}_{N}\right),
$$

where $R_{N}$ is the density matrix. The particles are bosons of the mass $m$ ( ${ }^{4} \mathrm{He}$ atoms) with their coordinates $\mathbf{r}_{i}$ restricted in volume $V$ being a cube with the periodic boundary conditions. In this case $R_{N}$ is given by [15]

$$
\begin{aligned}
& R_{N}\left(\mathbf{r}_{1}, \ldots, \mathbf{r}_{N} \mid \mathbf{r}_{1}^{\prime}, \ldots, \mathbf{r}_{N}^{\prime}\right)= \\
& \quad=P_{N}\left(\mathbf{r}_{1}, \ldots, \mathbf{r}_{N}\right) \frac{1}{N !} \frac{1}{\lambda^{3 N}} \sum_{Q} \exp \left(-\frac{\pi}{\lambda^{2}} \sum_{j=1}^{N}\left(\mathbf{r}_{j}-\mathbf{r}_{Q_{j}}^{\prime}\right)^{2}\right),
\end{aligned}
$$

where $Q$ is counting permutations of $(1, \ldots, N)$ and $\lambda$ is the thermal de Broglie wavelength

$$
\lambda=\left(\frac{2 \pi \beta \hbar^{2}}{m}\right)^{1 / 2} .
$$

The factor $P_{N}$ takes into account the interaction and will be described further.

The free energy $F_{N}$ is given by

$$
F_{N}=-\frac{1}{\beta} \ln Z_{N}
$$

total energy

$$
E_{N}=\frac{\partial}{\partial \beta}\left(\beta F_{N}\right)
$$


and heat capacity

$$
C_{N}=\beta^{2}\left[\frac{Z_{N}^{\prime \prime}}{Z_{N}}-\left(\frac{Z_{N}^{\prime}}{Z_{N}}\right)^{2}\right]
$$

the primes mean derivation over $\beta$.

\section{Partition function for the system of free particles}

The ideal Bose-gas is a well-studied system for which the expressions for the thermodynamic functions are known. Thus, it can be a good test example for the applicability of our method and we are going to use this fact here.

On calculating the thermodynamic functions of the ideal Bose-system with a small number of particles and comparing them with the well-known results for infinite system one can detect some regularities showing the point when several particles start to demonstrate the statistical behaviour.

One can find an expression for the partition function of $N$ ideal bosons in the explicit form. For this purpose we write the density matrix $R_{N}$ as follows:

$$
R_{N}\left(\mathbf{r}_{1}, \ldots, \mathbf{r}_{N} \mid \mathbf{r}_{1}, \ldots, \mathbf{r}_{N}\right)=\frac{1}{N !} \frac{1}{\lambda^{3 N}} \Delta_{N}
$$

where

$$
\Delta_{N}=\left|\begin{array}{ccccc}
1 & K_{12} & K_{13} & \ldots & K_{1 N} \\
K_{21} & 1 & K_{23} & \ldots & K_{2 N} \\
\vdots & & \ddots & & \\
K_{N 1} & K_{N 2} & K_{N 3} & \ldots & 1
\end{array}\right|_{+}
$$

and

$$
K_{i j} \equiv K\left(r_{i j}\right)=\mathrm{e}^{-\pi r_{i j}^{2} / \lambda^{2}}, \quad r_{i j}=\left|\mathbf{r}_{i j}\right|, \quad \mathbf{r}_{i j}=\mathbf{r}_{i}-\mathbf{r}_{j}
$$

The subscript "+" means that every "-" in the expression for the determinant should be substituted with "+".

The partition function for free particles is

$$
Z_{N}=\frac{1}{N !} \frac{1}{\lambda^{3 N}} \int \mathrm{d} \mathbf{r}_{1} \ldots \int \mathrm{d} \mathbf{r}_{N} \Delta_{N}
$$

The "determinant" (8) might be written as [15]

$$
\begin{aligned}
\Delta_{N}= & \Delta_{N-1}^{(1)}+\sum_{1<i \leqslant N} K_{1 i} K_{i 1} \Delta_{N-2}^{(1, j)} \\
& +\sum_{1<i<j \leqslant N}\left(K_{1 i} K_{i j} K_{j 1}+K_{1 j} K_{j i} K_{i 1}\right) \Delta_{N-3}^{(1, i, j)}+\ldots
\end{aligned}
$$


The superscripts in $\Delta_{N-l}$ mean the lack of the correspondent rows and columns in the expression for $\Delta_{N-l}$.

Integrating (10) we obtain

$$
\begin{aligned}
Z_{N}= & \frac{1}{N}\left\{\frac{V}{\lambda^{3}} Z_{N-1}+\frac{1}{\left(\lambda^{3}\right)^{2}} Z_{N-2} \int \mathrm{d} \mathbf{r}_{1} \int \mathrm{d} \mathbf{r}_{2} K_{12} K_{21}\right. \\
& \left.+\frac{1}{\left(\lambda^{3}\right)^{3}} Z_{N-3} \int \mathrm{d} \mathbf{r}_{1} \int \mathrm{d} \mathbf{r}_{2} \int \mathrm{d} \mathbf{r}_{3} K_{12} K_{23} K_{31}+\ldots\right\},
\end{aligned}
$$

After the Fourier transformation

$$
\begin{aligned}
K(r) & =\frac{1}{V} \sum_{\mathbf{q}} \mathrm{e}^{-i \mathbf{q r}} K_{\mathbf{q}} \\
K_{\mathbf{q}} & =\int \mathrm{d} \mathbf{r} \mathrm{e}^{i \mathbf{q r}} K(r)=\lambda^{3} \mathrm{e}^{-\lambda^{2} q^{2} / 4 \pi}
\end{aligned}
$$

the partition function is

$$
Z_{N}=\frac{1}{\rho \lambda^{3}} \sum_{l=1}^{N} \frac{Z_{N-l}}{l^{3 / 2}}, \quad Z_{0} \equiv 1
$$

One can use this expression to calculate the partition function of the system. We will make such calculations in the next section.

\section{Main results for the free particles}

It is possible to calculate heat capacities $C_{N}$ for $N=1,2,3, \ldots$ particles sequentially using expressions (6) and (15). In addition, we can show explicitly the low- and high-temperature asymptotics for $C_{N}$, namely:

$$
\begin{aligned}
& \left.\frac{C_{N}}{N}\right|_{T \rightarrow \infty}=\frac{3}{2} \\
& \left.\frac{C_{N}}{N}\right|_{T \rightarrow 0}=\frac{3}{2} \frac{1}{N}+A_{N} T^{3 / 2}, \quad A_{N}=\text { const with respect to } T .
\end{aligned}
$$

In the case of $N=\infty$ these temperature dependencies coincide with the known results for the ideal Bose-gas [16]. For low temperatures one has

$$
\left.\frac{C_{N}}{N}\right|_{N=\infty}=\frac{15}{4} \frac{\zeta(5 / 2)}{\zeta(3 / 2)}\left(\frac{T}{T_{\mathrm{c}}}\right)^{3 / 2} ;
$$

the high-temperature limit being $3 / 2$. The critical temperature $T_{\mathrm{c}}$ (the non-analyticity point on the heat capacity curve) in this case is defined from the equation

$$
\rho \lambda_{\mathrm{c}}^{3}=\zeta(3 / 2),
$$


Table 1. Main results for the ideal systems

\begin{tabular}{|c|c|c||c|c|c|}
\hline \hline$N$ & $\rho\left[\lambda_{\mathrm{c}}^{(N)}\right]^{3}$ & $C_{N}^{\max } / N$ & $N$ & $\rho\left[\lambda_{\mathrm{c}}^{(N)}\right]^{3}$ & $C_{N}^{\max } / N$ \\
\hline \hline 1 & - & 1.500 & 90 & 2.185 & 1.747 \\
\hline 2 & 1.131 & 1.532 & 100 & 2.200 & 1.753 \\
\hline 3 & 1.297 & 1.553 & 200 & 2.286 & 1.783 \\
\hline 5 & 1.490 & 1.582 & 300 & 2.328 & 1.799 \\
\hline 7 & 1.606 & 1.602 & 400 & 2.354 & 1.809 \\
\hline 10 & 1.717 & 1.624 & 500 & 2.373 & 1.816 \\
\hline 15 & 1.830 & 1.649 & 600 & 2.387 & 1.822 \\
\hline 20 & 1.902 & 1.666 & 700 & 2.399 & 1.827 \\
\hline 30 & 1.993 & 1.690 & 800 & 2.408 & 1.831 \\
\hline 40 & 2.050 & 1.706 & 900 & 2.416 & 1.834 \\
\hline 50 & 2.091 & 1.718 & 1000 & 2.423 & 1.837 \\
\hline 60 & 2.123 & 1.727 & 1100 & 2.429 & 1.839 \\
\hline 70 & 2.147 & 1.735 & 1200 & 2.434 & 1.842 \\
\hline 80 & 2.168 & 1.742 & $\infty$ & 2.612 & 1.926 \\
\hline \hline
\end{tabular}

where $\lambda_{\mathrm{c}}$ is the thermal de Broglie wavelength for $T_{\mathrm{c}}$, and $\zeta$ is the Rieman $\zeta$-function, $\zeta(3 / 2)=2.612 \ldots$ Taking $\rho=0.02185 \AA^{-3}$ for liquid ${ }^{4}$ He we obtain $T_{\mathrm{c}}=3.138 \mathrm{~K}$.

In this work we consider the systems with different $N$ from 2 to 1200 . As was mentioned above it is impossible to obtain the non-analyticity in the heat capacity, so we take the point of $C_{N}$ maximum as the critical temperature $T_{\mathrm{c}}^{(N)}$.

It appears that those values could be described by the linear dependence

$$
\rho\left[\lambda_{\mathrm{c}}^{(N)}\right]^{3} \simeq 2.61-\frac{c}{N^{1 / 3}}, \quad c \simeq 1.9
$$

with $\lambda_{\mathrm{c}}^{(N)}$ being thermal de Broglie wavelength for $T_{\mathrm{c}}^{(N)}$. One can easily notice that 2.61 is very close to $\zeta(3 / 2)=2.612 \ldots$.

We also have a similar correlation for the $C_{N}$ maxima $C_{N}^{\max } / N$. So, one should expect that the limit $N \rightarrow \infty$ gives the correct curve (see figures 1 and 2).

The results are also given in table 1 .

As one can see from figure 1, good qualitative and satisfactory quantitative agreement is reached at $N=100$. Although, typical elements of the heat capacity curve appear at smaller $N$, e. g., a clearly defined maximum is found at $N=10-15$. As we shall show further, these very values might be considered as the limit between "individual" and statistic behaviour of the system with $N$ particles. 


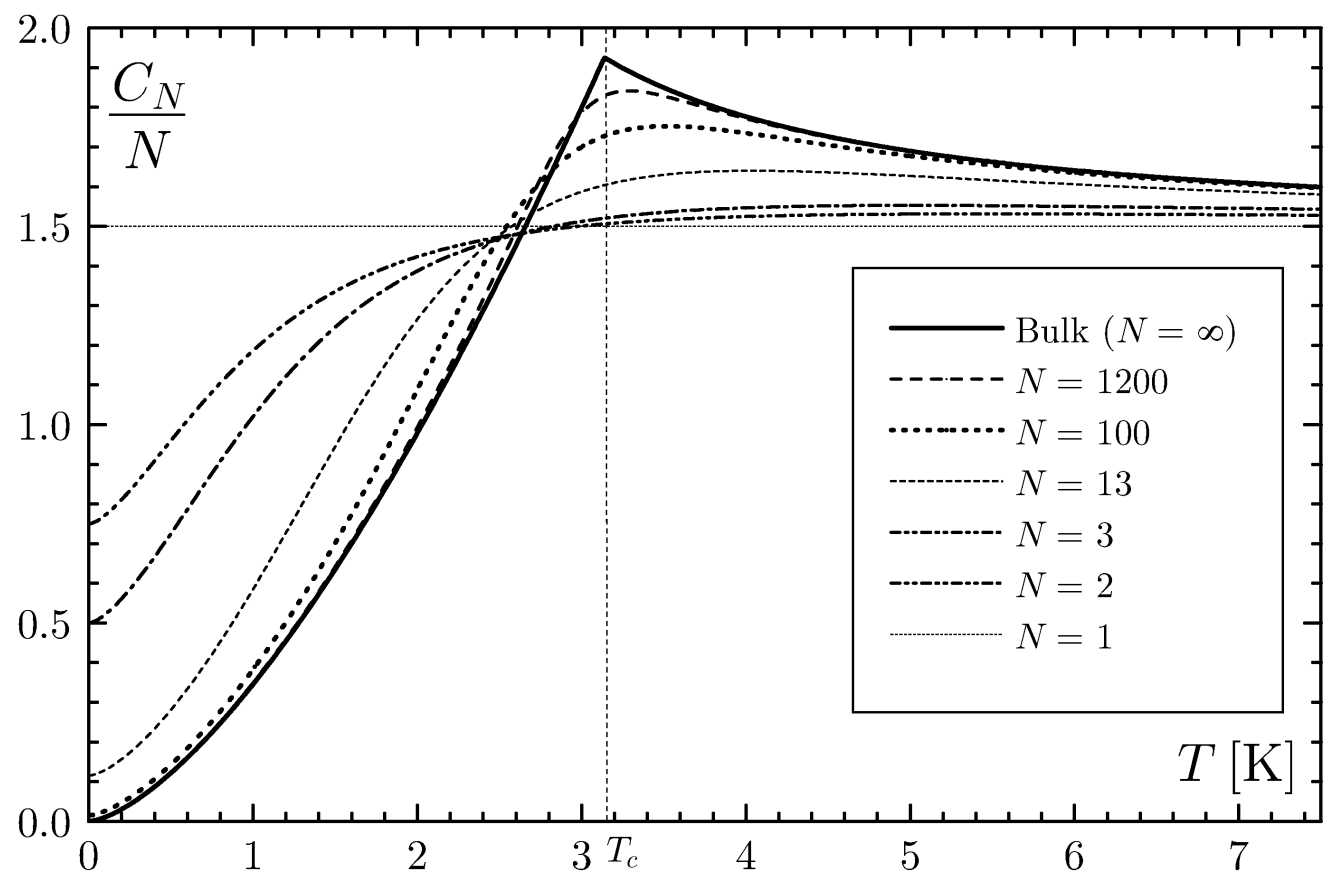

Figure 1. Heat capacities in the ideal case.

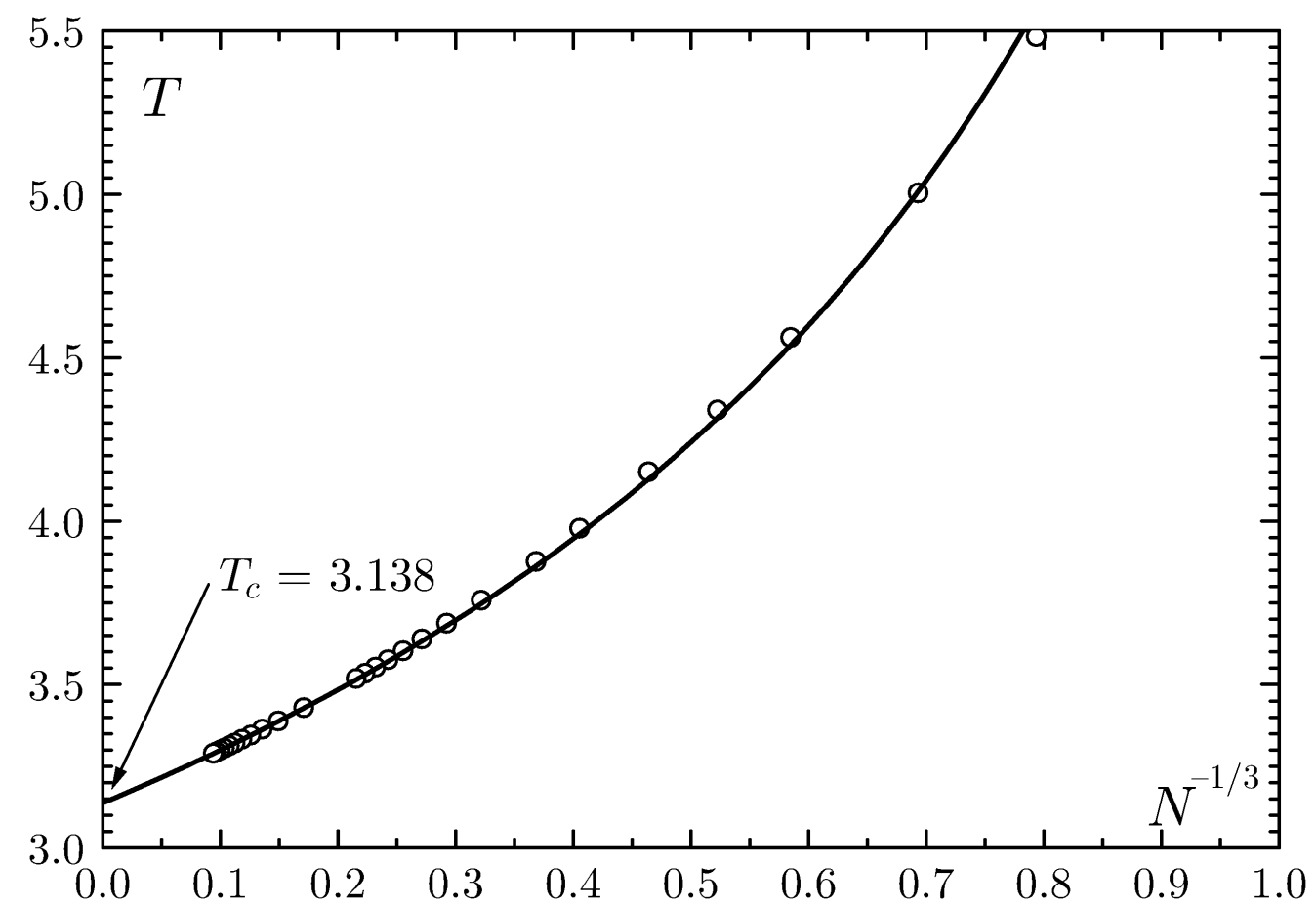

Figure 2. Temperature of the $C_{N} / N$ maxima in the ideal case. Solid line shows dependence (20), circles correspond to the data from table 1. 


\section{Interaction in the hard-spheres potential approximation}

In a many-body system, it is a very complicated problem to take into account the interaction and it has not as yet been completely solved. Specifically, the systems with a small number of particles make it possible to consider this problem from the first principles, i. e., to include the interaction into the calculations explicitly. From the mathematical point of view, the computations become heavily complicated while the number of particles increases, and the direct interaction account soon turns out to be impossible.

We will proceed from the expression for the density matrix $R_{N}$ in the free particles case. In the systems like ${ }^{4} \mathrm{He}$ small interatomic distances are essential when atoms behave almost as hard spheres. Thus, the wavefunction should vanish rapidly as soon as the distance between any two atoms approaches the hard sphere diameter.

Due to this we shall take the interaction into account by the substitution: of $\Delta_{N}$ in (10) with $\Delta_{N} P_{N}$ where $P_{N}$ is a function of the particles coordinates:

$$
Z_{N}=\frac{1}{N !} \frac{1}{\lambda^{3 N}} \int \mathrm{d} \mathbf{r}_{1} \ldots \int \mathrm{d} \mathbf{r}_{N} \Delta_{N} P_{N}
$$

For the sake of simplicity we suppose the interaction to have a pair nature since the obtained results are expected to be rather qualitative than quantitative. In the case of hard spheres potential we have

$$
\begin{aligned}
& P_{N}\left(\mathbf{r}_{1}, \ldots, \mathbf{r}_{N}\right)=\prod_{i=1}^{N-1} \prod_{j=2}^{N} P(i, j), \\
& P(i, j)=P\left(\left|\mathbf{r}_{i}-\mathbf{r}_{j}\right|\right)=P\left(r_{i j}\right), \\
& P(i, j)=1+f(i, j)=\left\{\begin{array}{ll}
0, & r_{i j} \leqslant a \\
1, & r_{i j}>a
\end{array},\right.
\end{aligned}
$$

$a$ is the hard sphere diameter.

One can write the partition function as

$$
\begin{gathered}
Z_{N}=\frac{1}{\rho \lambda^{3}} \sum_{l=1}^{N} b_{l}^{\prime}(N) Z_{N-l}, \quad Z_{0} \equiv 1, \\
\lambda=\left(\frac{2 \pi \beta \hbar^{2}}{m^{*}}\right)^{1 / 2},
\end{gathered}
$$

where $m^{*}$ is the effective mass of ${ }^{4} \mathrm{He}$ atom, $m^{*} \simeq 1.7 m[17]$.

We integrate (21) in two steps. The first one is the circular approximation (CA) and the second one is the free-volume approximation ( $F V A)$.

1) $\mathrm{CA}$ is to the effect that only the interparticle interactions in the circle $1-2-$ $3-\ldots-N-1$ are taken into account. It means we neglect the interaction between the first and the third, fourth, etc. particles, between the second and fourth, fifth, etc. 
particles, ... . This makes it possible to integrate (21) using Fourier transformation as we did in (10). Using also FVA we obtain for the coefficients $b_{l}^{\prime}(N)$ :

$$
b_{l}^{\prime}(N)=\left\{\begin{array}{cc}
{\left[1-\frac{v_{0}}{V}(N-l)\right]^{2}\left[1-\frac{v_{0}}{V}(N-3)\right] \prod_{k=3}^{l-1}\left[1-\frac{v_{0}}{V}(N-k)\right] I_{l-1},} & l>3 \\
{\left[1-\frac{v_{0}}{V}(N-l)\right]^{l} I_{l-1},} & l \leqslant 3
\end{array} .\right.
$$

Here we have used the following designations:

$$
\begin{gathered}
v_{0}=\frac{4}{3} \pi a^{3}, \\
I_{n}=\left\{\begin{array}{cc}
8(4 \pi)^{n-1} \int_{0}^{\infty} \mathrm{d} Q Q^{2}\left(\frac{1}{4 \pi} \mathrm{e}^{-Q^{2} / 4 \pi}-\frac{1}{Q} \int_{0}^{a / \lambda} x \sin Q x \mathrm{e}^{-\pi x^{2}} \mathrm{~d} x\right)^{n+1}, & n>0 \\
1, & n=0
\end{array}\right.
\end{gathered}
$$

2) In FVA we indirectly take into account the interactions neglected in CA. For this purpose each integral over $\mathbf{r}_{i}, i=1, \ldots, l$ is multiplied by the factor $\left(1-n v_{0} / V\right)$ where $n$ equals the number of multipliers $P(i, j)$ which are not taken into account in the Fourier transformation. To clarify the above statement we consider the most simple non-trivial example with $N=4$. According to (25) we have:

- $b_{1}^{\prime}(4)=\left[1-\frac{3 v_{0}}{V}\right] I_{0}$. Integral over $\mathbf{r}_{1}$ "hooks" the following three multipliers: $P(1,2), P(1,3), P(1,4)$.

- $b_{2}^{\prime}(4)=\left[1-\frac{2 v_{0}}{V}\right]^{2} I_{1}$. Integral over $\mathbf{r}_{1}$ "hooks" $P(1,2), P(1,3), P(1,4)$ but $P(1,2)$ is included into $I_{1}$. Integral over $\mathbf{r}_{2}$ "hooks" more $P(2,3)$ and $P(2,4)$.

- $b_{3}^{\prime}(4)=\left[1-\frac{v_{0}}{V}\right]^{3} I_{2}$. Each integral over $\mathbf{r}_{i}$ "hooks" additional $P(i, 2)$.

- $b_{4}^{\prime}(4)=\left[1-\frac{v_{0}}{V}\right]^{2} I_{3} . \quad P(1,3)$ and $P(2,4)$ are not included into $I_{3}$.

Thus, FVA improves the circular approximation. But it is not possible to use it for large $l$ and $N$ because in this case a big number of $P(i, j)$ multipliers will not be integrated in the proper way but via formal factors.

In the calculations we assume the hard sphere diameter $a=2.1 \AA$. The heat capacity curves exhibit a fast approaching of the maximum point to a value from the region of $2.2-2.3 \mathrm{~K}$ while $N$ is increasing. The exact $\lambda$-transition temperature is $2.17 \mathrm{~K}$. 


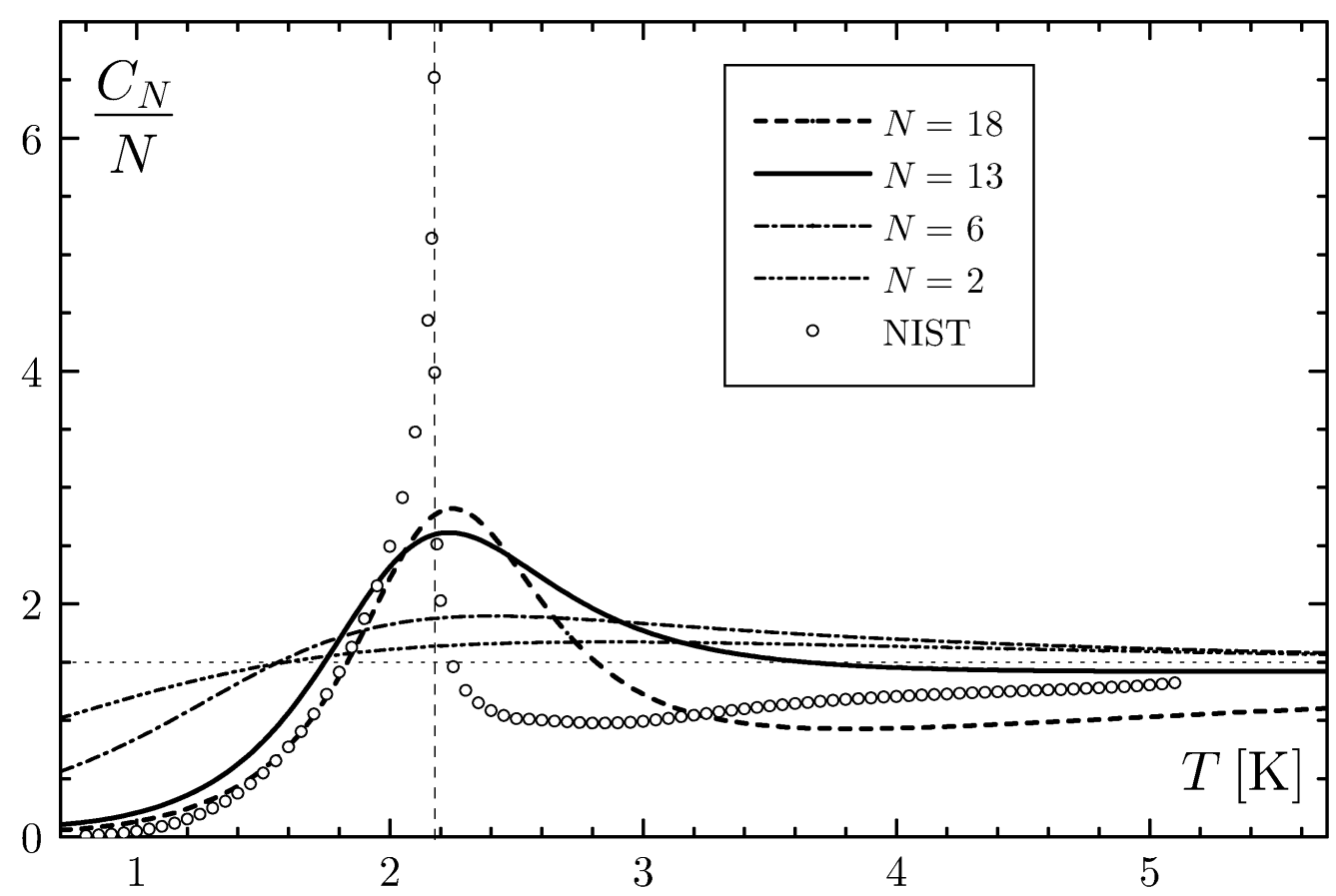

Figure 3. Heat capacities in the free volume and circular approximation. The circles show the NIST $C_{V}$ data [18].

As has already been mentioned, in the ideal case the linear (over $N^{-\varepsilon}$ ) dependence fits the sequence of $C_{N} / N$ maxima quite well

$$
\frac{C_{N}^{\max }}{N} \simeq C_{\infty}^{\max }-a N^{-\varepsilon}, \quad \varepsilon=1 / 3
$$

Assuming the same form for the hard spheres system one can find $C_{\infty}^{\max }=13.6$ and $\varepsilon=0.0608$ making the maxima sequence as follows:

$$
\frac{C_{N}^{\max }}{N} \simeq 13.6-12.9 N^{-\varepsilon}, \quad \varepsilon=0.0608 .
$$

Although small $N$ values of 12,13 do not allow one to identify the behaviour at $N \rightarrow \infty$ we obtain the values of heat capacity maximum from the range of experimental data $[7,18,19]$.

One can write the exact expression for the partition function similar to that in the case of $\mathrm{CA}$ and FVA:

$$
Z_{N}=\frac{1}{\rho \lambda^{3}} \sum_{l=1}^{N} b_{l}^{*}(N) Z_{N-l}, \quad Z_{0} \equiv 1 .
$$

From expansion (11) and formula (21) we obtain (without approximations):

$$
\begin{aligned}
b_{l}^{*}(N)= & \frac{1}{V} \frac{1}{(N-l) !} \frac{1}{\left(\lambda^{3}\right)^{N-1}} \int \mathrm{d} \mathbf{r}_{1} \ldots \int \mathrm{d} \mathbf{r}_{l} K_{12} \ldots K_{l-1, l} K_{l 1} \\
& \times \prod_{i=1}^{l-1} \prod_{j=i+1}^{l} P(i, j) \mathcal{B}_{l N}(1, \ldots, l),
\end{aligned}
$$




$$
\begin{aligned}
\mathcal{B}_{l N}(1, \ldots, l) & =\int \mathrm{d} \mathbf{r}_{l+1} \ldots \int \mathrm{d} \mathbf{r}_{N} \frac{\Delta_{N-l}^{(1, \ldots, l)} P_{N-l}^{(1, \ldots, l)} \prod_{i=1}^{l} \prod_{j=l+1}^{N} P(i, j)}{Z_{N-l}}, \\
P_{N-l}^{(1, \ldots, l)} & =\prod_{i=l+1}^{N-1} \prod_{j=i+1}^{N} P(i, j) .
\end{aligned}
$$

We also introduce the following designations to simplify further records:

$$
\begin{aligned}
R(1,2) & =\int \mathrm{d} \mathbf{r}_{3} P(1,3) P(2,3), \\
\widetilde{K}_{i j} & =K_{i j} P(i, j) .
\end{aligned}
$$

One can write the coefficients $b_{l}^{*}(N)$ independently of the interatomic potential, i. e. for any $P_{N}$ :

$$
\begin{aligned}
b_{1}^{*}(1) & =1 \\
b_{1}^{*}(2) & =\frac{1}{V^{2}} \int \mathrm{d} \mathbf{r}_{1} \int \mathrm{d} \mathbf{r}_{2} P(1,2), \\
b_{1}^{*}(3) & =\frac{\int \mathrm{d} \mathbf{r}_{1} \int \mathrm{d} \mathbf{r}_{2} P(1,2) R(1,2)\left(1+K_{12} K_{21}\right)}{\int \mathrm{d} \mathbf{r}_{1} \int \mathrm{d} \mathbf{r}_{2} P(1,2)\left(1+K_{12} K_{21}\right)}, \\
b_{2}^{*}(2) & =\frac{1}{V} \frac{1}{\lambda^{3}} \int \mathrm{d} \mathbf{r}_{1} \int \mathrm{d} \mathbf{r}_{2} K_{12} K_{21} P(1,2), \\
b_{2}^{*}(3) & =\frac{1}{V^{2}} \frac{1}{\lambda^{3}} \int \mathrm{d} \mathbf{r}_{1} \int \mathrm{d} \mathbf{r}_{2} K_{12} K_{21} P(1,2) R(1,2), \\
b_{3}^{*}(3) & =\frac{1}{V} \frac{1}{\left(\lambda^{3}\right)^{2}} \int \mathrm{d} \mathbf{r}_{1} \int \mathrm{d} \mathbf{r}_{2} \int d \mathbf{r}_{3} \widetilde{K}_{12} \widetilde{K}_{23} \widetilde{K}_{31} .
\end{aligned}
$$

These expressions might be written via one-dimensional integrals:

$$
\begin{aligned}
b_{1}^{*}(2) & =\frac{4 \pi}{V} \int_{0}^{\infty} P\left(r_{12}\right) r_{12}^{2} \mathrm{~d} r_{12}, \\
b_{2}^{*}(2) & =\frac{4 \pi}{\lambda^{3}} \int_{0}^{\infty} K_{12}^{2} P\left(r_{12}\right) r_{12}^{2} \mathrm{~d} r_{12}, \\
R\left(r_{12}\right) & =\frac{8}{r_{12}} \int_{0}^{\infty} \frac{\mathrm{d} q}{q} \sin q r_{12}\left\{\int_{0}^{\infty} r P(r) \sin q r \mathrm{~d} r\right\}^{2}, \\
b_{3}^{*}(3) & =\frac{32 \pi}{\left(\lambda^{3}\right)^{2}} \int_{0}^{\infty} \frac{\mathrm{d} q}{q}\left\{\int_{0}^{\infty} K_{12} P\left(r_{12}\right) r_{12} \sin q r_{12} \mathrm{~d} r_{12}\right\}^{3}, \\
b_{2}^{*}(3) & =\frac{4 \pi}{V \lambda^{3}} \int_{0}^{\infty} K_{12}^{2} P\left(r_{12}\right) R\left(r_{12}\right) r_{12}^{2} \mathrm{~d} r_{12} .
\end{aligned}
$$

In the hard spheres approximation, the integration is made in a simple way. We obtain

$$
b_{1}^{*}(1)=1
$$




$$
\begin{aligned}
b_{1}^{*}(2)= & 1-\frac{v_{0}}{V}, \\
b_{2}^{*}(2)= & \frac{1}{2^{3 / 2}}\left\{1-\operatorname{erf}\left(\frac{a}{\lambda} \sqrt{2 \pi}\right)\right\}+\frac{a}{\lambda} \mathrm{e}^{-2 \pi a^{2} / \lambda^{2}} \\
b_{1}^{*}(3)= & \frac{V-3 v_{0}+\frac{81}{32} \frac{v_{0}^{2}}{V}+\lambda^{3} b_{2}^{*}(3)}{V b_{1}^{*}(2)+\lambda^{3} b_{2}^{*}(2)} \\
b_{2}^{*}(3)= & \frac{V-v_{0}}{V}\left\{\frac{a}{\lambda}\left(\mathrm{e}^{-2 \pi a^{2} / \lambda^{2}}-2 \mathrm{e}^{-8 \pi a^{2} / \lambda^{2}}\right)\right. \\
& \left.+\frac{1}{2^{3 / 2}}\left(\operatorname{erf}\left(2 \frac{a}{\lambda} \sqrt{2 \pi}\right)-\operatorname{erf}\left(\frac{a}{\lambda} \sqrt{2 \pi}\right)\right)\right\} \\
& +\frac{\pi a^{2} \lambda}{V}\left\{\frac{a^{2}}{\lambda^{2}}\left(\mathrm{e}^{-2 \pi a^{2} / \lambda^{2}}-4 \mathrm{e}^{-8 \pi a^{2} / \lambda^{2}}\right)+\frac{1}{2 \pi}\left\{\mathrm{e}^{-2 \pi a^{2} / \lambda^{2}}\left(\frac{a^{4}}{\lambda^{4}}+\frac{1}{\pi} \frac{a^{2}}{\lambda^{2}}+\frac{1}{2 \pi^{2}}\right)\right.\right. \\
& \left.\quad-\frac{\left.\left.\mathrm{e}^{-2 \pi a^{2} / \lambda^{2}}-\mathrm{e}^{-8 \pi a^{2} / \lambda^{2}}\right)\right\}}{V-2 \lambda^{2}}\left(16 \frac{a^{4}}{\lambda^{4}}+\frac{4}{\pi} \frac{a^{2}}{\lambda^{2}}+\frac{1}{2 \pi^{2}}\right)\right\} \\
b_{3}^{*}(3)= & \left.32 \pi \int_{0}^{\infty} \frac{1}{2^{3 / 2}}\left(1-\operatorname{erf}\left(2 \frac{a}{\lambda} \sqrt{2 \pi}\right)\right)+2 \frac{a}{\lambda} \mathrm{e}^{-8 \pi a^{2} / \lambda^{2}}\right\} \\
& \left.\frac{V}{4 \pi} \mathrm{e}^{-Q^{2} / 4 \pi}-\int_{0}^{a / \lambda} x \sin Q x \mathrm{e}^{-\pi x^{2}} \mathrm{~d} x\right\}^{3}
\end{aligned}
$$

Our $R$ coincides with the corresponding function from [20]:

$$
R\left(r_{12}\right)=\int \mathrm{d} \mathbf{r}_{3}\left(1+f_{13}\right)\left(1+f_{23}\right)=V-2 v_{0}+g_{1}\left(r_{12}\right),
$$

The function $g_{1}\left(r_{12}\right)$ is given by

$$
g_{1}(r)=\int \mathrm{d} \mathbf{r}_{3} f_{13} f_{23}=\left\{\begin{array}{cc}
\frac{2}{3} \pi a^{3}\left(2-\frac{3}{2} \frac{r}{a}+\frac{1}{8}\left(\frac{r}{a}\right)^{3}\right), & r<2 a \\
0, & r \geqslant 2 a
\end{array} .\right.
$$

Meanwhile we cannot write exact expressions for greater $N$. The comparison of the exact results and those in CA and FVA is presented in table 2.

Our expressions for the partition function have the correct form $Z_{N}^{(\mathrm{cl})}$ in the classical limit $(\hbar \rightarrow 0)[21]$.

$$
Z_{N}^{(\mathrm{cl})}=\frac{1}{N !} \frac{1}{\lambda^{3 N / 2}} \int \mathrm{d} \mathbf{r}_{1} \ldots \int \mathrm{d} \mathbf{r}_{N} \prod_{1 \leqslant i<j \leqslant N} P(i, j) .
$$

It means that the proposed method is applicable to a wide temperature range.

\section{Conclusion}

It is shown that the proposed method leads to a good agreement between the calculated results and the experimental data. We obtained not only qualitative but even satisfactory quantitative fit as well. 
Table 2. Results for the interacting systems

\begin{tabular}{|c|c|c|c|c|}
\hline \multirow[b]{2}{*}{$N$} & \multicolumn{2}{|c|}{ Approximation } & \multicolumn{2}{|c|}{ Exact values } \\
\hline & $T_{\mathrm{c}}^{(N)}$ & $C_{N}^{\max } / N$ & $T_{\mathrm{c}}^{(N)}$ & $C_{N}^{\max } / N$ \\
\hline 1 & - & 1.50 & - & 1.50 \\
\hline 2 & 2.86 & 1.67 & 2.86 & 1.67 \\
\hline 3 & 2.54 & 1.78 & 2.19 & 1.83 \\
\hline 4 & 2.28 & 1.90 & - & - \\
\hline 5 & 2.25 & 2.01 & - & - \\
\hline 6 & 2.24 & 2.11 & - & - \\
\hline 7 & 2.23 & 2.20 & - & - \\
\hline 8 & 2.23 & 2.29 & - & - \\
\hline 9 & 2.23 & 2.37 & - & - \\
\hline 10 & 2.23 & 2.44 & - & $\overline{-}$ \\
\hline 11 & 2.23 & 2.50 & - & - \\
\hline 12 & 2.23 & 2.56 & - & - \\
\hline $13^{*}$ & 2.23 & 2.61 & - & - \\
\hline 18 & 2.23 & 2.82 & - & - \\
\hline$\infty$ & $2.2-2.3$ & $13.6^{* *}$ & - & - \\
\hline
\end{tabular}

* We suppose that the system of 12-13 particles behaves almost as a bulk since the atoms in ${ }^{4} \mathrm{He}$ are packed compactly enough to say that one atom is surrounded by 12 atoms. Therefore, 13 atoms constitute the first closed sphere (see figure 5).

** The experimental value is $\simeq 16$. The heat capacity in the $\lambda$-point was believed to be infinite for a long time but recent experiments show the finite peak [7]. 


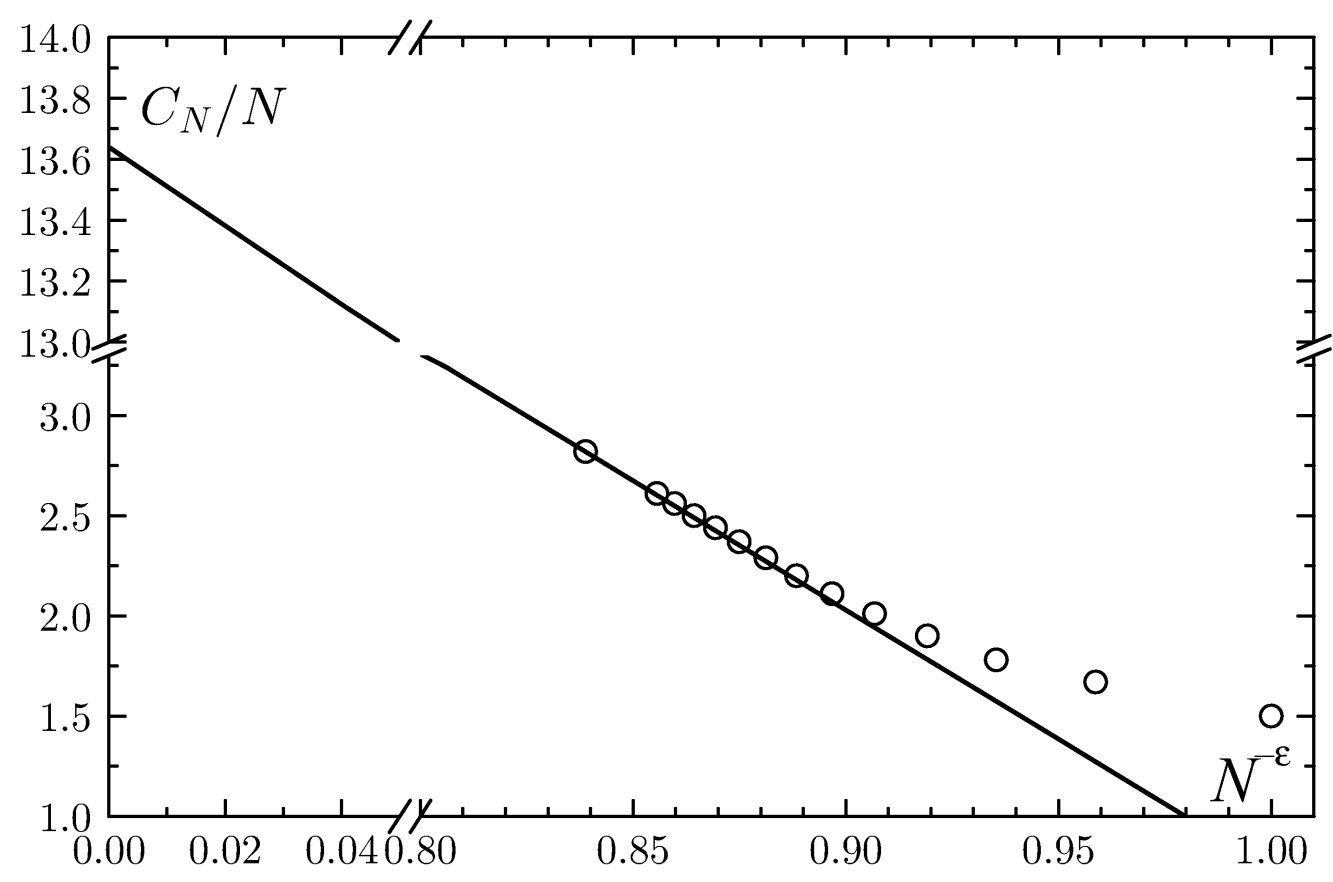

Figure 4. $C_{N}^{\max } / N$ values for the hard spheres system. Solid line shows dependence (29), circles correspond to the data from table 2 .

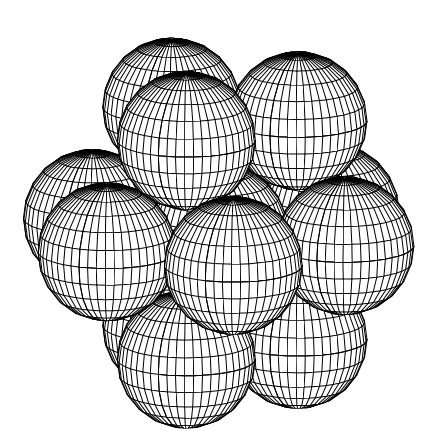

(a)

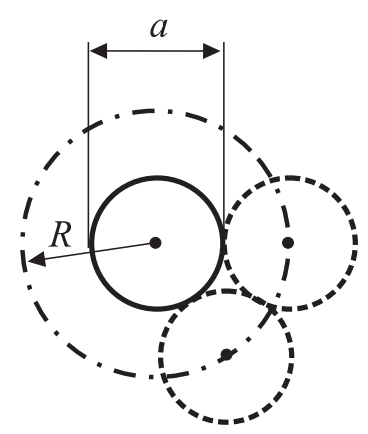

(b)

Figure 5. Helium- 4 atoms as hard spheres. (a) The first closed sphere of 12 atoms surrounding 1 central atom. One atom is not seen since it is situated exactly behind the central atom. (b) Solid line shows the "surface of atom", dashed line shows the "surface of alien atoms", dashed-dotted line shows "surface of inaccessible space". Thus, assuming the volume of "inaccessible space" $4 \pi R^{3} / 3$ to be the volume occupied by 1 atom one can obtain the hard sphere diameter $a \simeq 2.2 \AA$. 
The expansions over $1 / N$ obtained in this work demonstrate the existence of some parameter $\varepsilon$ having the value of $1 / 3$ for the ideal system. We estimated $\varepsilon=$ 0.0608 from the numerical analysis of the results. The theoretical calculation of this parameter will be our next problem along this line of research.

\section{Acknowledgements}

The authors appreciate Dr. Daniel G. Friend's help with the helium-4 thermophysical data.

\section{References}

1. Bogoliubov N.N. // J. Phys. (USSR), 1947, vol. 9, p. 23.

2. Brout R. // Phys. Rev., 1963, vol. 131, p. 899.

3. Feynman R.P. Statistical Mechanics. Mass., W. A. Benjamin, 1972.

4. Feynman R.P. // Phys. Rev., 1953, vol. 91, p. 1291, p. 1301.

5. Langer I.S. // Phys. Rev., 1968, vol. 167, p. 183.

6. Vakarchuk I.A. // Teor. Mat. Fiz., 1978, vol. 35, p. 76; Teor. Mat. Fiz., 1978, vol. 36, p. 122 (in Russian); Theor. Math. Phys., 1978, vol. 35, p. 327.

7. Lipa J.A., Swanson D.R., Nissen J.A. // Phys. Rev. Lett., 1996, vol. 76, p. 944.

8. Kleinert H. // Phys. Rev. D, 1999, vol. 60, p. 085001.

9. Kleinert H. // preprint cond-mat/9906107.

10. Campostrini M., Pelissetto A., Rossi P., Vicari E. // Phys. Rev. B, 2000, vol. 61, p. 5905.

11. Ceperley D.M. // Rev. Mod. Phys., 1995, vol. 67, p. 279.

12. Deng Wenji, Hui P.M. // Solid State Communications, 1997, vol. 104, p. 797.

13. Schmidt H.-J., Schnack J. // Physica A, 1998, vol. 260, p. 479.

14. Borrmann P., Franke G. // J. Chem. Phys., 1993, vol. 98, p. 2484.

15. Vakarchuk I.O. // J. Phys. Stud. (Lviv), 1996, vol. 1, p. 25 (in Ukrainian); J. Phys. Stud. (Lviv), 1997, vol. 1, p. 156 (in Ukrainian).

16. Huang K. Statistical Mechanics. New-York, Wiley, 1987.

17. Vakarchuk I.O. // Visn. Lviv. un-tu, Ser. fiz., 1993, vol. 26, p. 29 (in Ukrainian).

18. Arp V.D., McCarty R.D., Friend D.G. // Natl. Inst. Stand. Technol., Tech. Note 1334 (revised), 1998.

19. Coleman M., Lipa J.A. // Phys. Rev. Lett., 1995, vol. 74, p. 286.

20. Nijboer B.R.A., Van Hove L. // Phys. Rev., 1952, vol. 85, p. 777.

21. Isihara A. Statistical Physics. New York, Academic Press, 1971. 


\section{Термодинаміка Бозе-системи з малим числом частинок}

\section{І.О.Вакарчук, А.А.Ровенчак}

Кафедра теоретичної фізики, Львівський національний університет імені Івана Франка, 79005 Львів, вул. Драгоманова, 12

Отримано 28 травня 2001 р.

Запропоновано теоретичний опис взаємодіючої Бозе-системи, який ґрунтується на екстраполяції результатів, отриманих для систем 3 малим числом частинок $N=2,3,4$ і т.д. на випадок $N=\infty$. Показано, що вже система з $N=12,13$ поводиться майже як безмежна у широкому температурному діапазоні. Приділено особливу увагу фазовим переходам у цих системах. В обчисленнях використано потенціал твердих сфер. Послідовність максимумів теплоємности апроксимовано як $C_{N}^{\max } / N \simeq 13.6-a N^{-\varepsilon}$ з $\varepsilon=0.0608$, що дає для теплоємности безмежного зразка значення 13.6, експериментальне значення близьке до 16. Температуру $\lambda$-переходу оцінено як 2.1-2.3 K (експеримент дає 2.17 K). Досягнуто цілком добре якісне і задовільне кількісне узгодження з експериментальними даними.

Ключові слова: бозонні системи, скінчені системи, лямбда-перехід, питома теплоємність

PACS: 05.30.Jp, 74.25.Bt 
